\title{
Randomized comparison of 3 different-sized biopsy forceps for quality of sampling in Barrett's esophagus
}

\author{
Susana Gonzalez, MD, Woojin M. Yu, MD, Michael S. Smith, MD, MBA, Kristen N. Slack, BS, \\ Heidrun Rotterdam, MD, Julian A. Abrams, MD, MS, and Charles J. Lightdale, MD \\ Philadelphia, Hershey, Pennsylvania; New York, New York, USA
}

\begin{abstract}
Background-Several types of forceps are available for use in sampling Barrett's esophagus (BE). Few data exist with regard to biopsy quality for histologic assessment.

Objective-To evaluate sampling quality of 3 different forceps in patients with BE.

Design-Single-center, randomized clinical trial.

Patients-Consecutive patients with BE undergoing upper endoscopy.

Interventions-Patients randomized to have biopsy specimens taken with 1 of 3 types of forceps: standard, large capacity, or jumbo.
\end{abstract}

Main Outcome Measurements-Specimen adequacy was defined a priori as a well-oriented biopsy sample $2 \mathrm{~mm}$ or greater in diameter and with at least muscularis mucosa present.

\begin{abstract}
Results-A total of 65 patients were enrolled and analyzed (standard forceps, $\mathrm{n}=21$; largecapacity forceps, $\mathrm{n}=21$; jumbo forceps, $\mathrm{n}=23$ ). Compared with jumbo forceps, a significantly higher proportion of biopsy samples with large-capacity forceps were adequate $(37.8 \%$ vs $25.2 \%$, $P=.002)$. Of the standard forceps biopsy samples, $31.9 \%$ were adequate, which was not significantly different from specimens taken with large-capacity $(P=.20)$ or jumbo $(P=.09)$ forceps. Biopsy specimens taken with jumbo forceps had the largest diameter (median, $3.0 \mathrm{~mm}$ vs $2.5 \mathrm{~mm}$ [standard] vs $2.8 \mathrm{~mm}$ [large capacity]; $P=.0001)$. However, jumbo forceps had the lowest proportion of specimens that were well oriented (overall $P=.001$ ).
\end{abstract}

Limitations-Heterogeneous patient population precluded dysplasia detection analyses.

Conclusions-Our results challenge the requirement of jumbo forceps and therapeutic endoscopes to properly perform the Seattle protocol. We found that standard and large-capacity forceps used with standard upper endoscopes produced biopsy samples at least as adequate as those obtained with jumbo forceps and therapeutic endoscopes in patients with BE.

Copyright $@ 2010$ by the American Society for Gastrointestinal Endoscopy

Reprint requests: Charles J. Lightdale, MD, 180 Fort Washington Avenue, Harkness Pavilion 7-712, New York, NY 10032. Current affiliations: Departments of Medicine (S.G., J.A.A., C.J.L.) and Pathology (W.M.Y., H.R.), Columbia University Medical Center, New York, New York, Department of Medicine (M.S.S.), Temple University School of Medicine, Philadelphia, Pennsylvania, Department of Medicine (K.N.S.), Penn State College of Medicine Hershey, Pennsylvania.

Presented at American College of Gastroenterology Annual Scientific Meeting, October 23-28, 2009 (Am J Gastroenterol 2009; 104:S66).

DISCLOSURE: Dr. Abrams is supported in part by a K07 award from the National Cancer Institute (CA132892). Boston Scientific provided the forceps used in this study. Boston Scientific did not provide any financial support and did not participate in any other aspects of the study, including design, recruitment, data analysis, or manuscript preparation. All authors disclosed no financial relationships relevant to this publication. 
In Barrett's esophagus (BE), the normal squamous epithelium that lines the distal esophagus is replaced by columnar epithelium with specialized intestinal metaplasia. ${ }^{1,2}$ This metaplastic change is thought to be a consequence of longstanding gastroduodenal reflux, with an estimated prevalence of $1 \%$ to $5 \% .{ }^{3-5}$ Patients with the specialized columnar epithelium of BE are at increased risk of dysplasia and the development of adenocarcinoma of the esophagus. ${ }^{6,7}$

Current GI Society guidelines for the management of BE recommend surveillance endoscopy with random biopsy sampling to assess for the presence of dysplasia or adenocarcinoma ${ }^{8-10}$ The Seattle biopsy protocol is a widely accepted method for performing surveillance biopsies. ${ }^{11,12}$ This method involves the use of jumbo forceps with a therapeutic upper endoscope, application of the "turn-and-suck" technique, and random 4quadrant biopsies every 1 to $2 \mathrm{~cm}$ of the Barrett's segment. Previous studies have often used the diameter of the biopsy specimen as a surrogate for quality of sampling. However, proper interpretation of BE biopsy specimens by a pathologist requires that the sample also be properly oriented and at least to the depth of the muscularis mucosa. Currently, there are several biopsy forceps available for use with varying diameters, but there are limited data as to which forceps size is superior for obtaining optimal tissue samples.

Previous studies have compared various forceps to determine whether design influences tissue adequacy in sampling for GI diseases. ${ }^{13-15}$ For BE surveillance, the choice has traditionally been between standard forceps used with a standard upper endoscope or jumbo forceps used with a therapeutic upper endoscope. Large-capacity forceps are now available and have a larger outer diameter than standard forceps, but can easily be used with a standard endoscope. We performed a randomized trial to compare these newer largecapacity forceps with jumbo forceps and standard forceps with regard to technical quality of tissue sampling in patients with BE.

\section{METHODS}

\section{Study site}

This prospective, single-center study was performed from May to October 2008. All patients were being followed by 1 of 2 investigators with expertise in the management of BE (C.J.L. and J.A.A.).

\section{Patient selection}

Patients were eligible for participation in the study if they had a previous diagnosis of BE, defined as endoscopically suspected BE confirmed histologically by the presence of intestinal metaplasia. Any patient with a history of BE presenting for upper endoscopy was eligible, regardless of whether he or she had previously undergone endoscopic therapy (such as mucosal resection or radiofrequency ablation). The only exclusion criterion was the inability to give informed consent. One patient who was enrolled in the study was subsequently excluded; this patient was already enrolled in a separate trial in which biopsy samples were sent to an outside pathologist for review (and therefore not available to our study pathologists). The study was approved by the Institutional Review Board at Columbia University.

\section{Endoscopy and biopsies}

Before starting the endoscopy, patients were randomly assigned by sealed opaque envelopes that contained the forceps assignment to have biopsy samples taken with 1 of 3 biopsy forceps (Boston Scientific, Natick, Mass): standard forceps (Radial Jaw 3, 2.2-mm outer diameter), large-capacity forceps (Radial Jaw 4 Large Capacity, 2.4-mm outer diameter), or 
jumbo forceps (Radial Jaw 4 Jumbo, 2.8-mm outer diameter). The study coordinator opened the envelopes immediately before the procedures to determine which forceps would be used for the procedure. All patients underwent upper endoscopy and were examined with white light. Olympus Q180 upper endoscopes (Olympus Medical Systems, Center Valley, Pa) without high-definition capability were used for procedures with the standard and largecapacity forceps. Olympus T160 therapeutic endoscopes were used for all endoscopies performed with the jumbo forceps. Random 4-quadrant biopsy samples were taken for every 1 to $2 \mathrm{~cm}$ of $\mathrm{BE}$ for the entire length of BE identified. There was no visible BE in many of the patients who had previously undergone endoscopic therapy. In these cases, 4-quadrant random biopsy specimens were taken from the gastroesophageal junction. Biopsy samples were obtained by the turn-and-suction technique previously described. ${ }^{16}$ Any identified nodules or focal mucosal irregularities had targeted biopsies performed and samples submitted in a separate specimen container.

According to routine diagnostic procedures, the biopsy tissues were formalin fixed and processed for paraffin embedding. Biopsy specimens were not mounted before immersion fixation. Five-micron tissue sections were cut and stained with hematoxylin and eosin for histopathologic evaluation.

\section{Pathologic assessment}

Two expert GI pathologists who were blinded to the type of forceps used reviewed all the specimens. For each biopsy specimen, the following data were measured or assessed: greatest diameter, deepest tissue layer present (epithelium, lamina propria, muscularis mucosa, or sub-mucosa), and proper specimen orientation (yes or no). Proper orientation was defined as having 2 or more tissue layers in correct depth order in linear fashion. For instance, tangentially cut fragments or tissue fragments with surrounding epithelium were not considered to be properly oriented. The overall histology for each patient was recorded, with each patient categorized based on the highest degree of dysplasia on any biopsy (no BE, BE without dysplasia, low-grade dysplasia, high-grade dysplasia, or adenocarcinoma).

\section{Outcomes}

The primary outcome of the study was adequacy for histologic assessment. The a priori definition of an adequate biopsy sample was a well-oriented specimen $2 \mathrm{~mm}$ or greater in diameter with at least muscularis mucosa present. Secondary outcomes included comparisons of the individual measures: mean diameter, proportion of pieces $2 \mathrm{~mm}$ or greater, proportion of pieces with at least muscularis mucosa present, and proportion of pieces well oriented. Although the study was not designed to assess histologic endpoints, we also analyzed the proportion of patients with any dysplasia (low grade or greater), among patients with no previous treatment and no nodules or focal mucosal irregularities.

\section{Statistical analysis}

For comparison of the primary and secondary study outcomes, the 3 forceps were first compared by performing overall Fisher exact tests for categorical data, analysis of variance for normally distributed continuous data, or Kruskal-Wallis tests for non-normally distributed continuous data. Normality was assessed by the Skewness-Kurtosis test. If the overall $P$ value was <.05, then pairwise comparisons were performed by using the Fisher exact test and Wilcoxon rank-sum test. Bonferroni correction was performed to account for multiple comparisons $(\mathrm{n}=3)$ and thus statistical significance for these pairwise comparisons was defined as $P<.0167$. Otherwise, statistical significance was defined as $P<.05$. Twenty-two large-capacity forceps were provided for the study by Boston Scientific. Based on this number, we could potentially determine a $14 \%$ absolute difference in the primary 
outcome between any 2 forceps. All analyses were performed by using Stata 9.2 (StataCorp, College Station, Tex).

\section{RESULTS}

A total of 65 patients were enrolled in the study and included in the analyses. The mean age was 68.3 years (standard deviation 9.7), and $73 \%$ of patients were male (Table 1). The mean BE length was $1.7 \mathrm{~cm}$, and $55 \%$ of patients had received previous treatment for BE. A total of 206 biopsy samples were taken with standard forceps, 217 with large-capacity forceps, and 338 with jumbo forceps. There were no complications of the biopsies in any of the subjects.

Overall, 233 of 762 biopsies (30.6\%) were deemed adequate (Fig. 1). There was a significant overall difference in the proportion of biopsy samples that were adequate for histologic assessment (Table 2). A higher proportion of biopsy samples from large-capacity forceps were adequate compared with those taken with jumbo forceps $(37.8 \%$ vs $25.2 \%$, respectively; $P=.002$ ) (Fig. 2). There were no significant differences in the measure of adequacy between large-capacity and standard forceps and between standard and jumbo forceps.

A higher proportion of biopsy specimens were well oriented with both large-capacity forceps and standard forceps compared with the jumbo forceps (Table 2, PFig. 3). The diameter of biopsy samples taken with jumbo forceps was significantly greater than those taken with large-capacity forceps $(=.003)$ and standard forceps $(P=.0001)$ (Fig. 4). However, there was no overall difference among the 3 groups with regard to the proportion of biopsies $2 \mathrm{~mm}$ or greater or with muscularis mucosa present (Table 2).

Thirteen percent of the biopsy samples $(n=100)$ were taken from neosquamous epithelium, and $67 \%$ of these were from cases in which jumbo forceps were used. There was no statistically significant difference in diameter between biopsy specimens of neosquamous and Barrett's mucosa. However, a significantly lower proportion of neosquamous biopsy specimens were adequate $(P=.004)$ and well oriented $(P=.005)$ and contained at least muscularis mucosa $(P<.001)$. We therefore repeated the study analyses and stratified the results by neosquamous and columnar mucosa (Table 3 ). The results were qualitatively similar in the columnar mucosa samples. There was no significant difference in adequacy of the biopsy samples of neosquamous mucosa among the 3 biopsy forceps.

Twenty-six patients (40\%) had no previous endoscopic treatment and had no visible abnormalities seen on endoscopy. Among this group, dysplasia (low grade or greater) was detected in $9(34.6 \%)$. There was no significant difference in per-patient detection of dysplasia among the 3 forceps (standard, 30\%; large capacity, 50\%; jumbo, 25\%; overall $P$ value $=.67)$. No dysplasia was detected in any of the patients who had previously undergone endoscopic therapy.

\section{DISCUSSION}

The results of this randomized trial demonstrated that large-capacity forceps were superior to jumbo forceps with regard to specimen adequacy for pathologic assessment. Jumbo forceps produced the largest biopsy samples; however, a low proportion of these were well oriented compared with the standard and large-capacity forceps. For surveillance of BE, the use of jumbo forceps is frequently recommended with the assumption that the larger pieces will provide more Barrett's glands, which in turn would theoretically increase the detection of dysplasia. However, as demonstrated by the current study, larger specimens do not necessarily translate into superior sampling quality. 
Although jumbo forceps produced the largest biopsy samples, these forceps also had the lowest proportion of specimens deemed adequate for pathologic assessment. The reasons for these findings are not entirely clear. One possible explanation is that use of a therapeutic endoscope limits tip deflection within the esophagus, resulting in a more acute angle between the forceps and the esophageal wall. In fact, we found that biopsy samples obtained with large-capacity forceps (using a standard endoscope) were more likely to contain muscularis mucosa compared with jumbo forceps. It is also conceivable that jumbo forceps somehow have a greater shear effect, thereby producing greater artifact and limiting biopsy sample adequacy.

Use of large-capacity biopsy forceps is intriguing because they have a larger outer diameter compared with standard forceps, but can still be used with a standard upper endoscope (with a 2.8-mm working channel). Jumbo forceps can theoretically be used with a standard upper endoscope. However, suction cannot reliably be applied with the forceps in place, precluding use of the turn-and-suck technique. Anecdotally, we have accidentally used jumbo forceps with a standard upper endoscope and have found that significant force is required to retract the forceps into the channel. Additionally, it has been our experience that the newer standard upper endoscopes have markedly increased maneuverability compared with therapeutic upper endoscopes.

In a recent study in BE patients undergoing surveillance, jumbo forceps had significantly higher rates of adequate biopsy samples compared with standard forceps (79\% vs $16 \%$, respectively; $P<.05) .{ }^{14}$ Adequacy was defined as the presence of muscularis mucosa, minimal crush artifact, and proper fixation. Notably, specimen orientation was not part of the definition. In our study, a relatively low proportion of jumbo forceps biopsy specimens were well oriented, resulting in a lower overall adequacy rate. With regard to the presence of muscularis mucosa, jumbo forceps fared worse and standard forceps performed better than in the previously cited study. Reasons for these differences are unclear, but may include operator technique and use of different endoscopes. Our findings overall also demonstrated that biopsy specimens of neosquamous epithelium were less likely to be adequate compared with columnar mucosa. However, given the small number of neosquamous biopsy samples, no definitive conclusions can be drawn regarding the use of different biopsy forceps to detect subsquamous intestinal metaplasia in neosquamous mucosa.

The current study has several strengths. This was a randomized trial of 3 types of forceps, and the pathologists were blinded to the type of forceps used for each case. The primary outcome, adequacy for pathologic assessment, was defined a priori.

There are certain limitations to this study. Our aim was not to address dysplasia detection in BE surveillance, but to determine the technical aspects of performing biopsies with various forceps and which forceps would produce optimal results with regard to histologic assessment. We recognize that the most important clinical endpoint is the ability to detect dysplasia. We chose specimen adequacy as a surrogate measure of sampling quality, although this measure potentially may not correlate with dysplasia detection. Our study enrolled a heterogeneous group of patients, including 55\% of patients who had previously undergone endoscopic therapy. Additionally, we did not orient the biopsy specimens before fixation, as advocated by the Seattle protocol. However, we have observed that this additional step is rarely performed in clinical practice and is not routinely performed at our institution in the handling of esophageal biopsy specimens. Overall, $30.6 \%$ of biopsy samples were adequate in our study, which is quite low; however, our definition of adequacy has not previously been validated. Harrison et al ${ }^{17}$ previously demonstrated that even in patients with an endoscopically visible columnar-lined esophagus, intestinal metaplasia was demonstrated in only $64 \%$ of patients and only $34 \%$ of the individual biopsy samples 
contained foci of intestinal metaplasia. These low rates of detection have previously been argued as a need for more biopsies because of the heterogeneous nature of a columnar-lined esophagus. However, we speculate that it may also be because of inadequate sampling by different biopsy forceps and, therefore, tissue acquisition with forceps that provide adequate samples for analysis would be important in sampling for BE.

Our study did not address the use of high-definition white light endoscopy, narrow-band imaging, or other advanced imaging techniques in the detection of dysplasia. Use of these modalities to identify focal abnormalities for targeted biopsies may ultimately have a much greater impact on dysplasia detection than the choice of forceps for random surveillance biopsies in BE. Additionally, jumbo forceps optimally require a 3.2-mm working channel of a therapeutic upper endoscope, and there are currently no therapeutic upper endoscopes with both high-definition and narrow-band imaging capabilities.

The results of our randomized trial in patients with BE demonstrated that large-capacity forceps were more likely to produce biopsy samples that were adequate for histologic assessment compared with jumbo forceps. There was no significant difference between the large-capacity and standard forceps and standard and jumbo forceps with respect to the production of adequate biopsy specimens. Jumbo forceps produced the largest samples, although these samples were often not well oriented. There were no significant differences in per-patient dysplasia detection rates, although the study was not designed to address this particular question. However, our results challenge the stipulation that jumbo forceps and a therapeutic endoscope are essential requirements to obtain optimal results by using the Seattle protocol. Based on our findings, we conclude that standard and large-capacity forceps used with a standard upper endoscope produce biopsy samples in patients with BE that are at least as adequate as those using jumbo forceps with a therapeutic endoscope.

\title{
Abbreviation
}

\author{
BE Barrett's esophagus
}

\section{References}

1. Spechler SJ. Clinical practice. Barrett's esophagus. N Engl J Med. 2002; 346:836-42. [PubMed: 11893796]

2. Shaheen NJ, Richter JE. Barrett's oesophagus. Lancet. 2009; 373:850-61. [PubMed: 19269522]

3. Cameron AJ, Zinsmeister AR, Ballard DJ, et al. Prevalence of columnar-lined (Barrett's) esophagus. Comparison of population-based clinical and autopsy findings. Gastroenterology. 1990; 99:918-22. [PubMed: 2394347]

4. Ronkainen J, Aro P, Storskrubb T, et al. Prevalence of Barrett's esophagus in the general population: an endoscopic study. Gastroenterology. 2005; 129:1825-31. [PubMed: 16344051]

5. Conio M, Cameron AJ, Romero Y, et al. Secular trends in the epidemiology and outcome of Barrett's oesophagus in Olmsted County, Minnesota. Gut. 2001; 48:304-9. [PubMed: 11171817]

6. Morales CP, Souza RF, Spechler SJ. Hallmarks of cancer progression in Barrett's oesophagus. Lancet. 2002; 360:1587-9. [PubMed: 12443613]

7. Rastogi A, Puli S, El-Serag HB, et al. Incidence of esophageal adenocarcinoma in patients with Barrett's esophagus and high-grade dysplasia: a meta-analysis. Gastrointest Endosc. 2008; 67:3948. [PubMed: 18045592]

8. Hirota WK, Zuckerman MJ, Adler DG, et al. ASGE guideline: the role of endoscopy in the surveillance of premalignant conditions of the upper GI tract. Gastrointest Endosc. 2006; 63:570 80. [PubMed: 16564854]

9. Wang KK, Sampliner RE. Updated guidelines 2008 for the diagnosis, surveillance and therapy of Barrett's esophagus. Am J Gastroenterol. 2008; 103:788-97. [PubMed: 18341497] 
10. Wang KK, Wongkeesong M, Buttar NS. American Gastroenterological Association technical review on the role of the gastroenterologist in the management of esophageal carcinoma. Gastroenterology. 2005; 128:1471-505. [PubMed: 15887129]

11. Reid BJ, Blount PL, Feng Z, et al. Optimizing endoscopic biopsy detection of early cancers in Barrett's high-grade dysplasia. Am J Gastroenterol. 2000; 95:3089-96. [PubMed: 11095322]

12. Levine DS, Haggitt RC, Blount PL, et al. An endoscopic biopsy protocol can differentiate highgrade dysplasia from early adenocarcinoma in Barrett's esophagus. Gastroenterology. 1993; 105:40-50. [PubMed: 8514061]

13. Dolwani S, Saleem H, Thompson IW, et al. A comparison of three types of biopsy forceps in the endoscopic surveillance of Barrett's oesophagus. Endoscopy. 2002; 34:946-9. [PubMed: 12471536]

14. Komanduri S, Swanson G, Keefer L, et al. Use of a new jumbo forceps improves tissue acquisition of Barrett's esophagus surveillance biopsies. Gastrointest Endosc. 2009; 70:172-8.

15. Elmunzer BJ, Higgins PD, Kwon YM, et al. Jumbo forceps are superior to standard large-capacity forceps in obtaining diagnostically adequate inflammatory bowel disease surveillance biopsy specimens. Gastrointest Endosc. 2008; 68:273-8. quiz 334, 336. [PubMed: 18155204]

16. Levine DS, Reid BJ. Endoscopic biopsy technique for acquiring larger mucosal samples. Gastrointest Endosc. 1991; 37:332-7. [PubMed: 2070985]

17. Harrison R, Perry I, Haddadin W, et al. Detection of intestinal metaplasia in Barrett's esophagus: an observational comparator study suggests the need for a minimum of eight biopsies. Am $\mathbf{J}$ Gastroenterol. 2007; 102:1154-61. [PubMed: 17433019] 


\section{Take-home Message}

- Large-capacity and standard forceps were more likely than jumbo forceps to produce biopsy specimens that were adequate for histologic assessment. These results challenge the requirement of jumbo forceps and therapeutic endoscopes in Barrett's esophagus surveillance. 


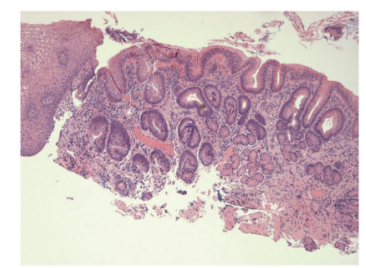

Figure 1.

Photomicrograph of an adequate biopsy sample, defined as a diameter of $2 \mathrm{~mm}$ or greater, properly oriented, and containing at least muscularis mucosa (H\&E, orig. mag. $\times 4)$. 


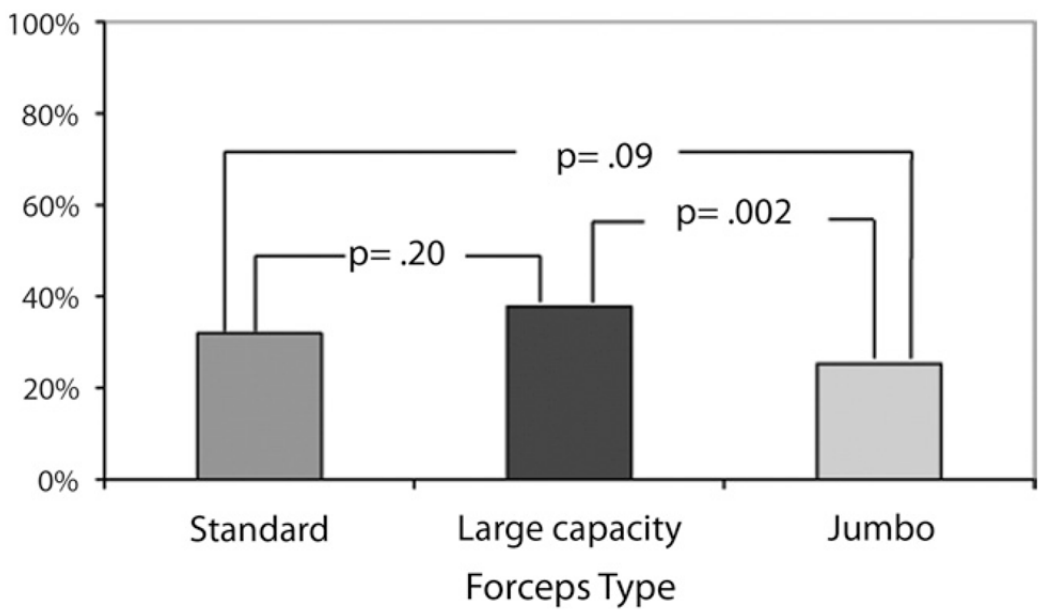

Figure 2.

Proportions of biopsy samples adequate for pathologic assessment in patients with BE compared among standard, large-capacity, and jumbo forceps. 


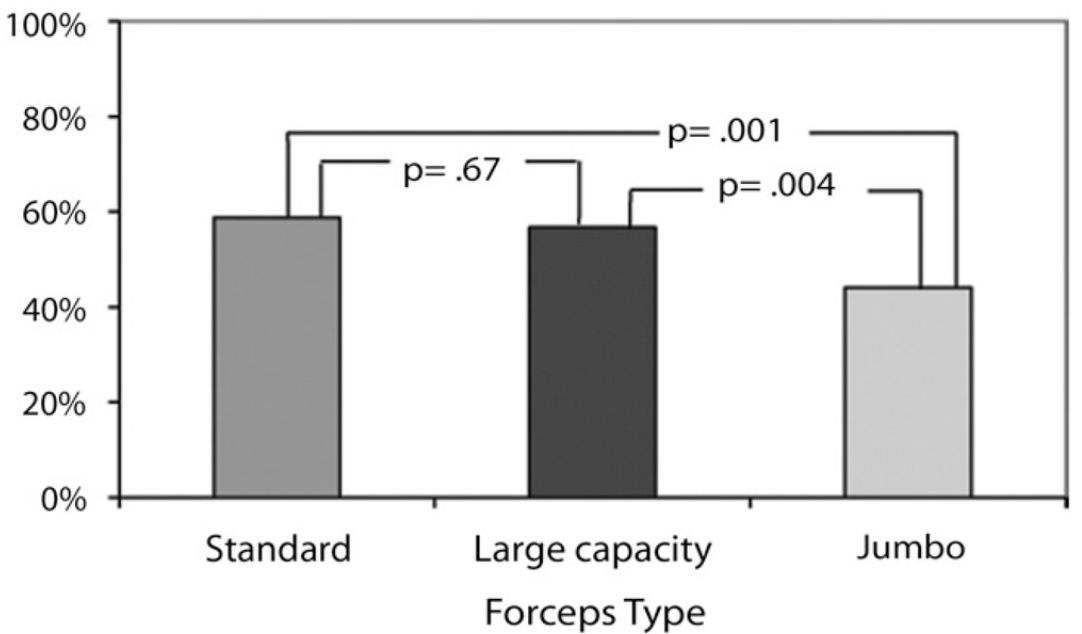

Figure 3.

Proportions of biopsy samples properly oriented in patients with BE compared among standard, large-capacity, and jumbo forceps. 


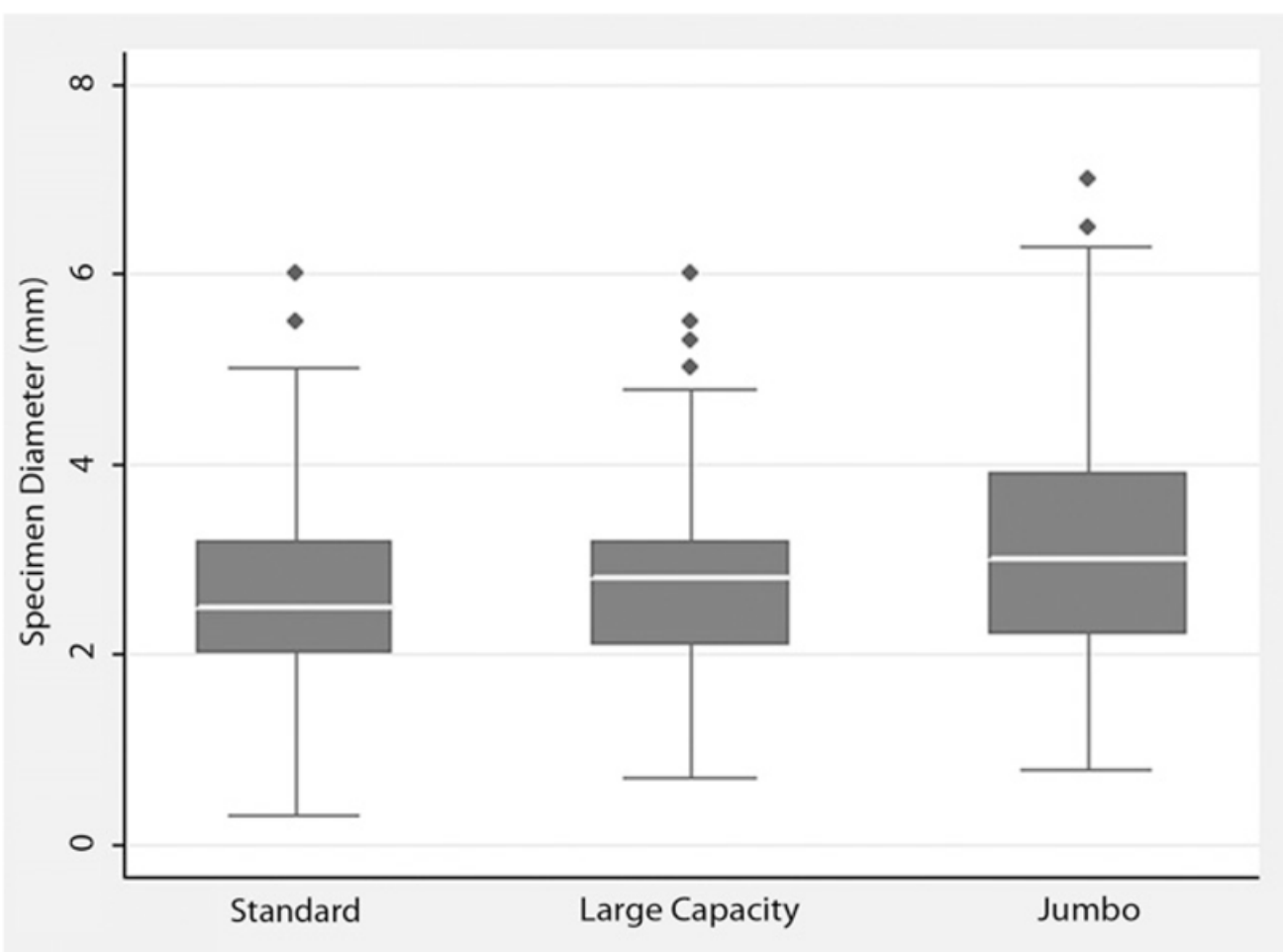

Figure 4.

Median diameter (with interquartile range) of biopsy samples from patients with BE compared among standard, large-capacity, and jumbo forceps. Biopsy specimens taken with jumbo forceps had a significantly larger diameter than those obtained with large-capacity $(P$ $=.003)$ and standard $(P=.0001)$ forceps. There was no difference in the diameter of samples obtained with standard and large-capacity forceps $(P=.19)$. 
TABLE 1

Patient demographics and endoscopic characteristics

\begin{tabular}{|lcccc|}
\hline & Standard & Large capacity & Jumbo & $\boldsymbol{P}$ value \\
\hline Total & 21 & 21 & 23 & \\
\hline Mean (SD) age (y) & $70.5(9.3)$ & $70(7.7)$ & $65(11)$ & .11 \\
\hline Males, no. (\%) & $14(67)$ & $17(81)$ & $16(70)$ & .55 \\
\hline Mean BE length (cm) & 1.7 & 1.3 & 2.1 & .66 \\
\hline Nodule or mass, no. (\%) & $1(5)$ & $1(5)$ & $2(9)$ & 1.00 \\
\hline Previous BE treatment, no. (\%) & $10(45)$ & $12(57)$ & $13(57)$ & .78 \\
\hline
\end{tabular}

$B E$, Barrett's esophagus; $S D$, standard deviation. 
TABLE 2

Results of histopathologic assessment of biopsy specimens from patients with Barrett's esophagus comparing standard, large-capacity, and jumbo forceps

\begin{tabular}{|lcccc|}
\hline & Standard & Large capacity & Jumbo & $\boldsymbol{P}$ value \\
\hline No. of gross specimens & 206 & 217 & 338 & \\
\hline Adequate biopsy specimens (\%) & 31.9 & 37.8 & 25.2 & .006 \\
\hline Median greatest diameter, mm & 2.5 & 2.8 & 3.0 & .0001 \\
\hline Specimens $\geq 2 \mathrm{~mm}(\%)$ & 80.6 & 83.9 & 87.3 & .11 \\
\hline Specimens with at least muscularis mucosa (\%) & 40.3 & 44.2 & 36.1 & .13 \\
\hline Specimens well oriented $(\%)$ & 58.7 & 56.7 & 44.1 & .001 \\
\hline
\end{tabular}


TABLE 3

Stratified analysis of neosquamous and columnar biopsy samples adequate for pathologic assessment compared among standard, large-capacity and jumbo forceps

\begin{tabular}{|lcccc|}
\hline & Standard & Large capacity & Jumbo & $\boldsymbol{P}$ value \\
\hline Columnar mucosa & & & & .06 \\
\hline Total no. of samples & 197 & 194 & 271 & \\
\hline \% Adequate & 33 & 38 & 28 & \\
\hline Neosquamous mucosa & & & & .10 \\
\hline Total no. of samples & 10 & 23 & 67 & \\
\hline \% Adequate & 10 & 35 & 15 & \\
\hline
\end{tabular}

